\title{
An Arabidopsis gene expression predictor enables inference of transcriptional regulators
}

Haiying Geng ${ }^{{ }^{*}}$, Meng Wang ${ }^{* 1}$, Jiazhen Gong ${ }^{* 1}$, Yupu $\mathrm{Xu}^{1}$, Shisong $\mathrm{Ma}^{1,2}$

1. Hefei National Laboratory for Physical Sciences at the Microscale, School of Life Sciences, Division of Life Sciences and Medicine, University of Science and Technology of China, Innovative Academy of Seed Design, Chinese Academy of Sciences, Hefei, China

2. School of Data Science, University of Science and Technology of China, Hefei, China

* These authors contribute equally.

Correspondence: Shisong Ma (sma@ustc.edu.cn)

\begin{abstract}
Gene expression regulation by transcription factors (TF) has long been studied, but no model exists yet that can accurately predict transcriptome profiles based on TF activities. We have constructed a universal predictor for Arabidopsis to predict the expression of 28192 non-TF genes using 1678 TFs. Applied to bulk RNA-Seq samples from diverse tissues, the predictor produced accurate predicted transcriptomes correlating well with actual expression, with average correlation coefficient of 0.986 . Having recapitulated the quantitative relationships between TFs and target genes, the predictor further enabled downstream inference of TF regulators for genes and pathways, i.e. those involved in suberin, flavonoid, glucosinolate metabolism, lateral root, xylem, secondary cell wall development, and endoplasmic reticulum stress response. Our predictor provides an innovative approach to study transcriptional regulation.
\end{abstract}

\section{INTRODUCTION}

Transcriptome, a critical determinant of cellular functions and status, is largely modulated by gene transcription. Transcription in turn is mainly managed by a network of transcription factors (TF) that integrate environmental and developmental cues to fine tune target genes' expression $(1,2)$. Gene regulatory networks (GRN) that connect TFs to their target genes are key to decipher cellular functions and regulatory mechanisms (3). GRNs have been constructed systematically by characterizing TF binding motifs, by profiling TF-DNA interaction, or by perturbing TFs expression (4-7). They were also inferred from transcriptome data using computational approaches like probabilistic graphical model, neural network, 
LASSO regression, least angle regression, and decision tree (8-12). Nevertheless, for both plants and animals, comprehensive and universal models that quantitatively predict gene expression levels based on TF activities have yet to be built. Here, we describe an Arabidopsis gene expression predictor for reconstructing highly accurate transcriptome profiles based on TFs expression. The predictor is tissueindependent and can be universally applied to samples derived from all tissues. It also enables downstream inference of TF regulators for genes and pathways, thus facilitating mechanistic investigation on transcriptional regulation.

\section{RESULTS AND DISCUSSION}

\section{A universal gene expression predictor for Arabidopsis}

We aimed to construct a predictor that predicts Arabidopsis gene expression values based on TFs' expression levels and use it to infer TF regulators for genes and pathways. A linear predictor model:

$$
Y=X B+\varepsilon
$$

was built via ordinary least squares regression, where target genes' expression matrix $Y$ were estimated as linear combinations of TFs' expression matrix $X$, with $B$ and $\varepsilon$ representing the coefficient matrix and random errors (Figure 1A). Large-scaled Arabidopsis RNA-Seq raw data were downloaded from NCBI's Sequence Read Archive (SRA) database and processed via a uniform pipeline. After quality filtering, transcriptomes from 24545 RNA-Seq runs derived from diverse tissues (Figure 1B) were kept and merged into a large expression matrix for predictor model training. The predictor, after excluding low-expressed genes, shall take $1678 \mathrm{TF}$ genes as inputs to predict the expression of 29182 non-TF genes.

To obtain a universal predictor, the sample diversity and sample number of the training dataset are critical. A predictor model trained with leaf samples only performed poorly in predicting transcriptomes from roots and seeds, with low Pearson's correlation coefficients $(r)$ and high normalized root mean square errors (NRMSE) between the predicted and actual gene expression values (Figure 1C). Adding root and seed transcriptomes to the training dataset improved predicting quality for independent root and seed samples, while, interestingly, also improved prediction for independent leaf samples. Thus synergism was observed that combining transcriptomes from different tissues in the training dataset yielded better models than using single tissue alone. Since our model has a large number of input TFs, over-fitting might be a concern. A model trained with 1700 samples, despite possessing very high $r(0.9999)$ for the training samples, had limited ability to predict independent test samples (with average test $r 0.6612$ and NRSME 1.0294), indicating high level of optimism or over-fitting within the model. As the number of training samples increased, the model's ability to predict test samples improved, and the difference between the 
training and test $r$ and NRSME decreased, suggesting the decline of over-fitting (Figure 1D). Indeed, a model trained with 20000 samples had average $r$ of 0.9926 and 0.9896 for the training and test samples, respectively. The model explained $98.52 \%\left(r^{2}\right)$ of the variances of gene expression values in the training samples, among which $0.6 \%$ might be due to over-fitting. Thus, increasing the number of training samples to $20000+$ reduced over-fitting to a negligible level.

In light of the above results, all transcriptomes of the 24545 RNA-Seq runs were used to train the predictor model. To evaluate the predictor's performance, RNAs were extracted from Arabidopsis root and shoot tissues to generate two RNA-Seq transcriptomes. Using the expression levels of $1678 \mathrm{TF}$ genes, the predictor accurately predicted the expression of other 29182 non-TF genes for these two samples, with $r>$ 0.994 and NRSME $<0.075$ (Figure 2A). A leave-one-out cross-validation (LOOCV) strategy was also employed to assess the predictor's ability to predict other novel independent samples. The 24545 RNA-Seq runs used for predictor training came from 1342 NCBI SRA studies. In each LOOCV run, a study was selected whose samples were held out, and the predictor was re-trained with all other RNA-Seq samples and then tested on the hold-out samples. This is similar to applying the predictor to novel independent samples. Figure 2B shows the LOOCV results for the study SRP075604 that had measured 138 transcriptomes across diverse Arabidopsis tissues (13). The predictor had highly accurate predictions for samples derived from all these tissues, with an average $r$ of 0.9874 , although the performance was slightly better for leaf and root samples than anther samples (Figure 2B). In total, LOOCV test was conducted for 546 studies that contain 10 or more RNA-Seq runs. The predictor had highly accurate predictions for samples from most of these studies, with average $r$ of $0.9861 \pm 0.0177$ and NRSME of $0.1066 \pm 0.0499$ (Figure 2C). However, the predictor had moderate performance on single-cell RNA-Seq samples (data not shown). Single-cell RNA-Seq samples accounted for only a minor fraction of all Arabidopsis RNA-Seq datasets and they were excluded from our analysis. Thus, we have constructed a highly accurate and tissueindependent gene expression predictor that can be applied universally to most Arabidopsis bulk RNA-Seq samples.

\section{Arabidopsis gene co-expression modules}

Besides the predictor model, gene co-expression modules were also identified using the same expression dataset. An Arabidopsis gene co-expression network based on the graphical Gaussian model (GGM) was constructed via a previously published procedure (14). The partial correlation coefficients (pcor) between all genes were calculated, and 312790 significant co-expressed gene pairs with pcor $>=$ 0.045 were extracted to build a gene co-expression network (Figure S1 and Table S1). The network was then clustered into 1085 gene co-expression modules via the Markov Clustering (MCL) algorithm (Figure 3A and Table S2) (15). According to guilt-by-association, genes within the same module have similar 
expression patterns and might function in the same pathways. Indeed, 378 identified modules have enriched gene ontology $(\mathrm{GO})$ terms $(P<=0.001)$ and function in pathways involved in, i.e., metabolism, development, and stress response (Table S3). For example, Module \#84 was considered as a suberin biosynthesis module since it is enriched with 6 suberin biosynthetic genes $(P=3.82 \mathrm{E}-11)$, like $C Y P 86 B 1$, $A B C G 20, A B C G 6, G P A T 5$, and RWP1 (Figure 3B). Module \#272 participates in glucosinolate biosynthesis $(P=9.04 \mathrm{E}-19)$ and contains key genes like CYP79B3, SUR1, and UGT74B1, while \#210 is involved in flavonoid and anthocyanin biosynthesis $(P=1.02 \mathrm{E}-17)$ with genes like TT3, TT18, At5MAT (Figure 3C, D). Development related modules were identified as well, such as \#105 for lateral root development $(P=$ $1.86 \mathrm{E}-06)$, \#65 for xylem development $(P=1.05 \mathrm{E}-14)$, and \#11 for secondary cell wall biogenesis $(P=$ $9.25 \mathrm{E}-43$ ) in the late stage of xylem formation (Figure $3 \mathrm{E}-\mathrm{G})$. Also recovered were modules involved in stress response, such as \#46 for endoplasmic reticulum (ER) stress response $(P=3.96 \mathrm{E}-50)$ (Figure $3 \mathrm{H}$ ). The genes within these modules shared similar expression patterns and might be regulated by the same set of TFs. It is of great interest to identify such TFs as they could be the master transcriptional regulators for pathways that define a plant's form and functions.

\section{Transcriptional regulators revealed by the predictor}

The predictor model and gene co-expression modules were then used to infer transcriptional regulators for genes and pathways. The predictor essentially recapitulated the quantitative relationships between TFs and target genes within its coefficient matrix $B$. Significance tests were conducted for individual regression coefficients $\beta$ within the matrix, and 980736 significant interacting TF-target gene pairs corresponding to the coefficients with p-value $<=1 \mathrm{E}-09$ (approximately equal to a Bonferronicorrected p-value of 0.05 ) were extracted (Table S4). The TFs connected to a target gene by these significant TF-gene pairs were then considered as that gene's predictor TFs. In average, each gene has 34 predictor TFs. These predictor TFs can be deemed as the gene's potential transcriptional regulators. As an example, 37 TFs were identified as predictor TFs for CYP86B1, a gene encoding a fatty acid cytochrome P450 oxidase required for suberin biosynthesis (Figure 4A) (16). Its top predictor TFs include MYB41, MYB107, MYB93, NAC058, and WRKY56. Among them, MYB41 and MYB107 are transcriptional regulators of suberin biosynthesis, whose over-expression or mutation altered the expression of CYP86B1, while MYB93's homologue from apple MdMyb93 also modulates suberin deposition (17-19). Thus, among the 37 predictor TFs for CYP86B1 include its known regulators and possibly other potential novel regulators as well.

Beyond individual genes, predictor TFs were also inferred at the pathways level for gene coexpression modules. As an example, Module \#84 for suberin biosynthesis contains 45 non-TF genes, including CYP86B1. These genes' common predictor TFs were then considered as the module's predictor 
TFs. For instance, MYB41 is shared by 42 non-TF genes within Module \#84 as their predictor TF, while it has only 281 target genes among all non-TF genes. Thus MYB4I's target genes are enriched 97 folds within the module $(P=1.16 \mathrm{E}-82)$. A TF gene was considered as a predictor TF for a module if it was shared by at least $20 \%$ or 6 , depending on which is larger, of the module's non-TF genes as predictor TF and its target genes were also enriched within the module $(P<=1 \mathrm{E}-06)$. Under such criterion, 27 TFs, including $M Y B 41$, were identified as predictor TFs for Module \#84, among which are known suberin regulators like MYB41, MYB107, MYB9 $(17,18)$, and other MYB-, NAC-, and WRKY-type TFs as potential regulators (Figure 4B). Interestingly, among the potential regulators, $M Y B 39$ was recently shown to be another regulator of suberization (20).

Predictor TFs were also identified for another 635 modules (Table S5). In average, 12 predictor TFs were identified for each module. Many known and potential novel TF regulators for diverse pathways were revealed. For example, predictor TFs were recovered for the development-related modules. Among them, Module \#105, involved in lateral root development, had 7 predictor TFs, LBD29, LBD18, LBD16, PUCHI, GATA23, LBD17, and WOX11, all of which except $L B D 17$ have been shown to be regulators of lateral root development (Figure 4C) $(21,22)$. Module \#65, for xylem development, had 10 predictor TFs, 7 of which are known modulators (MP, WOX4, ATHB8, TMO5, T5L1, VND5, ANT) of xylem or vascular development $(23,24)$ and the other 3 could be potential regulators (Figure 4D). On the other hand, all identified predictors for Module \#11, functioning in secondary cell wall biogenesis, are known regulators of secondary cell wall biosynthesis, including SND1, SND2, SND3, VND4, VND6, MYB46, MYB103, MYB83, IRX11, NST2, LBD15 and AtC3H14 (Figure 4E) (5, 25-28).

Also revealed were predictor TFs for modules functioning in metabolism and stress responses. For example, Module \#210, involved in flavonoid and anthocyanin biosynthesis, had 9 predictor TFs, including TT8, TTG2, PAP1, PAP2, GL3, MYB11, MYB113, MYBL2 and NF-YA10 (Figure 4F). Surprisingly, all of them except $N F-Y A 10$ have been shown as regulators of flavonoid/anthocyanin biosynthesis $(29,30)$. In contrast, Module \#272, participating in glucosinolate biosynthesis, had 22 predictor TFs, but only 7 of them (MYB28, MYB29, MYB76, MYB34, MYB51, MYB122, MYC4) have been shown to regulate glucosinolate biosynthesis (31-33), indicating there might be more potential regulators among the rest predictor TFs (Figure 4G). Similarly, the ER stress response module (\#46) had 50 identified predictor TFs, among which 6 are known ER stress regulators (bZIP60, bZIP17, bZIP28, NAC089, NAC062, TBF1) and they cover most of the major TFs regulating ER stress that have been characterized so far (34-36), while the rest 44 could be potential modulators (Figure $4 \mathrm{H}$ ).

Besides the examples mentioned above, modules and their predictor TFs were also identified for many other plant processes, such as the development of pollen tube (Module \#1), anther (\#78), Casparian 
strip (\#138), root (\#71), root hair (\#5), flower (\#132), shoot (\#102), the metabolism of wax (\#33), phenylpropanoid (\#61), fatty acid (\#47), nitrate (\#15), phosphate (\#38), sulfur (\#91), iron (\#58, 86, 222), and the response to heat (\#125), cold (\#170), water deprivation (\#224), hypoxia (\#35), and biotic stresses (\#12) (Table S3 and S5). These examples demonstrated that our approach can effectively identify gene modules associated with a broad range of biological processes and their known and potential transcriptional regulator TFs.

Overall, we have constructed a highly accurate and tissue-independent gene expression predictor to predict Arabidopsis transcriptomes based on TFs expression. The key to obtain such a universal predictor was using a large number of transcriptomes derived from diverse tissues to train the predictor. Our results indicated the high-dimensioned gene expression profiles can be modeled as linear combinations of reduceddimensioned TFs expression data, which is consistent with previous report that gene expression data have low dimensionality (37). There were also reports to use a small number of selected informative marker genes to impute gene expression profiles $(38,39)$. But these studies mainly focused on transcriptome prediction, without further mechanistic inference. In contrast, by using TFs as regressors to construct the model, our predictor not only achieved highly accurate predicted transcriptomes, but also recapitulated the quantitative relationships between TFs and target genes that seem to preserve across tissues, which further enabled downstream inference of TF regulators for genes and pathways. As demonstrated by the aforementioned examples, many of the identified predictor TFs for the modules are known regulators of the related pathways, while the rest could serve as novel potential regulators. The same approach, which we named as EXPLICIT (Expression Prediction via Linear Combination of Transcription Factors), also worked for other plant and animal species, such as rice, maize, mouse, and human (Wang et al. submitted, and unpublished data). Thus, for multiple eukaryotic species, there exist universal coefficient matrices that govern the quantitative relationships between TFs and target genes across tissues. These matrices provide the keys to understanding transcriptional regulation in biological systems.

\section{MATERIALS AND METHODS}

\section{Gene expression data and gene expression predictor model}

Raw data of publicly available Arabidopsis RNA-Seq runs were downloaded from NCBI SRA database. After removing single-cell or small RNAs sequencing runs and the runs with less than 1000000 reads, reads from the remaining runs were mapped against Arabidopsis genome (Araport11) using STAR 34 (version 020201) to produce read counts for each annotated genes (40). The cpm (counts per million) expression values were calculated for each gene, and the runs with unique mapping rates $<50 \%$ or total 
mapping rates $<70 \%$ or with $<5000$ genes having cpm $>=1$ were removed. Low-expressed genes that had $\mathrm{cpm}>=1$ in less than 100 samples were also filtered out. Finally, the expression values of 30860 genes in 24545 RNA-Seq runs from 1342 SRA studies were kept and merged into a large expression matrix after $\log$ transformation $\left(\log _{2}(\mathrm{cpm}+1)\right)$ for further analysis.

Using an Arabidopsis TF gene list obtained from PlantTFDB (41), two expression matrix for 1678 TF genes $X$ and 29182 non-TF genes $Y$ were extracted and used to train a gene expression predictor using a linear model $Y=X B+\varepsilon$, with $B$ and $\varepsilon$ representing coefficient matrix and random errors. The coefficient matrix was estimated in MATLAB (version R2019a) via least squares as $B=\left(X^{\prime} X\right) \backslash\left(X^{\prime} Y\right)$. The significance of individual regression coefficients within the predictor model were also determined via $t$ test, following hypothesis test method for multiple linear regression (42). The resulted p-values were used to extract significant interacting TF-target gene pairs from the predictor model. Predictor TFs for every nonTF gene were then identified as those TFs connected to the gene by these significant interacting TF-target gene pairs. A MATLAB script was developed in-house to conduct the analysis.

To test the predictor performance on transcriptome prediction, Arabidopsis RNA-Seq samples were processed via the same pipeline described above and two actual expression matrix for TFs $X_{t}$ and non-TFs $Y_{t}$ were extracted. A predicted expression matrix was estimated as $Y_{p}=X_{t} B . Y_{p}$ and $Y_{t}$ were then used to calculate the Pearson's correlation coefficients $r$ and normalized root mean squared errors (NRMSE) between the predicted and estimated transcriptomes. NRMSE for a given sample was calculated as:

$$
N R M S E=\sqrt{\frac{\sum_{i=1}^{n}\left(y_{i}-\tilde{y}_{i}\right)^{2}}{\sum_{i=1}^{n}\left(y_{i}\right)^{2}}}
$$

where $y_{i}$ and $\tilde{y}_{i}$ denote the actual and predicted expression values of gene $\mathrm{i}$ within the sample.

\section{Arabidopsis tissues and RNA-Seq sequencing}

Surface-sterilized Arabidopsis seeds were stratified at $4^{\circ} \mathrm{C}$ in dark for 2 days and placed on vertical $1 / 2$ MS Agar plates supplemented with $1 \%(\mathrm{w} / \mathrm{v})$ sucrose and $0.8 \%(\mathrm{w} / \mathrm{v})$ agar at $22{ }^{\circ} \mathrm{C}$ under $16 \mathrm{~h}$ day $/ 8 \mathrm{~h}$ night cycle for growing. After 14 days, root and shoot tissues were harvested separately and total RNAs were extracted using TRI Reagent (Sigma-Aldrich, St. Louis, MO, USA). The quantity and integrity of the total RNAs were assessed on an Agilent 2100 Bioanalyzer (Agilent Technologies, Palo Alto, CA, USA), and only the samples with RIN values $\geq 8$ were used for constructing mRNA sequencing libraries. mRNAseq libraries were prepared for sequencing using NEBNext ${ }^{\circledR}$ Ultra ${ }^{\mathrm{TM}}$ RNA Library Prep Kit for Illumina ${ }^{\circledR}$ (New England BioLabs, Ipswich, MA, USA) according to manufacturer's protocols. All libraries were 
sequenced using the Illumina NovaSeq platforms with paired-end 150 bp (PE 150) sequencing strategy. The library construction and sequencing were performed by the Novogene Corporation (Beijing, China).

\section{Gene module and predictor TF identification}

Using the expression matrix composed of 30860 genes in 24545 RNA-Seq runs, an Arabidopsis gene co-expression network based on the graphical Gaussian model was constructed via a procedure published previously (14). Briefly, a random sampling strategy was used to calculate the pcors between all gene pairs. The procedure consisted of 20000 rounds. In each round, 2000 genes were randomly selected and pcors between them were calculated using the GeneNet package in R (43). After 20000 rounds, each gene pairs were sampled 83 times with 83 pcors calculated, and the pcor with lowest absolute value was then chosen as that gene pair's final pcor. The gene pairs with $p c o r>=0.045$ were then chosen to construct a GGM gene co-expression network for Arabidopsis. The network was then clustered using the MCL algorithm (15), and 1085 gene modules that contained at least 6 genes were kept for further analysis. The network and its modules were visualized in Cytoscape (V3.4.0) (44). Arabidopsis GO terms annotations were obtained from TAIR (45), and GO enrichment analysis for the module were conducted using the hypergeometric distribution.

Predictor TFs were then identified for the modules. If a TF gene were shared by at least 6 as well as $20 \%$ of the non-TF genes within a module, and the TF's target gene were also enriched within the module as determined via the hypergeometric distribution, the TF was considered as a target gene for the module. A perl script was developed in-house to identify predictor TFs for modules. The predictor TFs and target genes' interactions within a module were visualized as Chord diagrams using the 'circlize' package in $\mathrm{R}$ (46).

\section{ACKNOWLEDGEMENT}

This work was supported by grants from the National Natural Science Foundation of China (31770268), the Strategic Priority Research Program of the Chinese Academy of Sciences (XDA24010302), the Fundamental Research Funds for the Central Universities (WK2070000091), and University of Science and Technology of China (Start-up fund to S.M.). The numerical calculations in this manuscript were conducted on the supercomputing systems in USTC Supercomputing Center and USTC School of Life Sciences Bioinformatics Center. 


\section{REFERENCES}

1. P. J. Mitchell, R. Tjian, Transcriptional regulation in mammalian cells by sequence-specific DNA binding proteins. Science 245, 371-378 (1989).

2. J. L. Riechmann et al., Arabidopsis transcription factors: Genome-wide comparative analysis among eukaryotes. Science 290, 2105-2110 (2000).

3. M. M. Babu, N. M. Luscombe, L. Aravind, M. Gerstein, S. A. Teichmann, Structure and evolution of transcriptional regulatory networks. Curr. Opin. Struct. Biol. 14, 283-291 (2004).

4. M. B. Gerstein et al., Architecture of the human regulatory network derived from ENCODE data. Nature 489, 91-100 (2012).

5. M. Taylor-Teeples et al., An Arabidopsis gene regulatory network for secondary cell wall synthesis. Nature 517, 571-575 (2015).

6. M. T. Weirauch et al., Determination and Inference of Eukaryotic Transcription Factor Sequence Specificity. Cell 158, 1431-1443 (2014).

7. Z. Hu, P. J. Killion, V. R. Iyer, Genetic reconstruction of a functional transcriptional regulatory network. Nat Genet 39, 683-687 (2007).

8. N. Friedman, Inferring cellular networks using probabilistic graphical models. Science 303, 799805 (2004).

9. A.-C. Haury, F. Mordelet, P. Vera-Licona, J.-P. Vert, TIGRESS: Trustful Inference of Gene REgulation using Stability Selection. BMC Systems Biology 6, 145 (2012).

10. N. Omranian, J. M. O. Eloundou-Mbebi, B. Mueller-Roeber, Z. Nikoloski, Gene regulatory network inference using fused LASSO on multiple data sets. Scientific reports 6, 14 (2016).

11. E. Segal et al., Module networks: identifying regulatory modules and their condition-specific regulators from gene expression data. Nat Genet 34, 166-176 (2003).

12. J. VohradskÝ, Neural network model of gene expression. The FASEB Journal 15, 846-854 (2001).

13. A. V. Klepikova, A. S. Kasianov, E. S. Gerasimov, M. D. Logacheva, A. A. Penin, A high resolution map of the Arabidopsis thaliana developmental transcriptome based on RNA-seq profiling. The Plant Journal 88, 1058-1070 (2016).

14. S. Ma, Q. Gong, H. J. Bohnert, An Arabidopsis gene network based on the graphical Gaussian model. Genome Res 17, 1614-1625 (2007).

15. A. J. Enright, S. Van Dongen, C. A. Ouzounis, An efficient algorithm for large-scale detection of protein families. Nucleic Acids Res 30, 1575-1584 (2002).

16. V. Compagnon et al., CYP86B1 is required for very long chain omega-hydroxyacid and alpha, omega -dicarboxylic acid synthesis in root and seed suberin polyester. Plant Physiol 150, 18311843 (2009).

17. J. Lashbrooke et al., MYB107 and MYB9 Homologs Regulate Suberin Deposition in Angiosperms. Plant Cell 28, 2097-2116 (2016).

18. D. K. Kosma et al., AtMYB41 activates ectopic suberin synthesis and assembly in multiple plant species and cell types. Plant J 80, 216-229 (2014).

19. S. Legay et al., MdMyb93 is a regulator of suberin deposition in russeted apple fruit skins. New Phytol 212, 977-991 (2016).

20. H. Cohen, V. Fedyuk, C. Wang, S. Wu, A. Aharoni, SUBERMAN regulates developmental suberization of the Arabidopsis root endodermis. Plant J, (2020).

21. J. A. S. Teixeira, K. H. T. Tusscher, The Systems Biology of Lateral Root Formation: Connecting the dots. Mol Plant, (2019).

22. L. Sheng et al., Non-canonical WOX11-mediated root branching contributes to plasticity in Arabidopsis root system architecture. Development 144, 3126-3133 (2017). 
23. H. Fukuda, K. Ohashi-Ito, Vascular tissue development in plants. Curr Top Dev Biol 131, 141-160 (2019).

24. R. S. Randall et al., AINTEGUMENTA and the D-type cyclin CYCD3;1 regulate root secondary growth and respond to cytokinins. Biol Open 4, 1229-1236 (2015).

25. R. Zhong, C. Lee, Z. H. Ye, Evolutionary conservation of the transcriptional network regulating secondary cell wall biosynthesis. Trends Plant Sci 15, 625-632 (2010).

26. J. Zhou, R. Zhong, Z. H. Ye, Arabidopsis NAC domain proteins, VND1 to VND5, are transcriptional regulators of secondary wall biosynthesis in vessels. PloS one 9, e105726 (2014).

27. K. Ohashi-Ito, K. Iwamoto, H. Fukuda, LOB DOMAIN-CONTAINING PROTEIN 15 Positively Regulates Expression of VND7, a Master Regulator of Tracheary Elements. Plant Cell Physiol 59, 989-996 (2018).

28. G. Chai et al., Arabidopsis $\mathrm{C} 3 \mathrm{H} 14$ and $\mathrm{C} 3 \mathrm{H} 15$ have overlapping roles in the regulation of secondary wall thickening and anther development. J Exp Bot 66, 2595-2609 (2015).

29. W. Xu, C. Dubos, L. Lepiniec, Transcriptional control of flavonoid biosynthesis by MYB-bHLH-WDR complexes. Trends Plant Sci 20, 176-185 (2015).

30. A. Gonzalez, M. Zhao, J. M. Leavitt, A. M. Lloyd, Regulation of the anthocyanin biosynthetic pathway by the TTG1/bHLH/Myb transcriptional complex in Arabidopsis seedlings. Plant J 53, 814827 (2008).

31. F. Schweizer et al., Arabidopsis basic helix-loop-helix transcription factors MYC2, MYC3, and MYC4 regulate glucosinolate biosynthesis, insect performance, and feeding behavior. Plant Cell 25, 3117-3132 (2013).

32. I. E. Sonderby, M. Burow, H. C. Rowe, D. J. Kliebenstein, B. A. Halkier, A complex interplay of three R2R3 MYB transcription factors determines the profile of aliphatic glucosinolates in Arabidopsis. Plant Physiol 153, 348-363 (2010).

33. H. Frerigmann, T. Gigolashvili, MYB34, MYB51, and MYB122 distinctly regulate indolic glucosinolate biosynthesis in Arabidopsis thaliana. Mol Plant 7, 814-828 (2014).

34. C. Ruberti, S.-J. Kim, G. Stefano, F. Brandizzi, Unfolded protein response in plants: one master, many questions. Current Opinion in Plant Biology 27, 59-66 (2015).

35. K. M. Pajerowska-Mukhtar et al., The HSF-like transcription factor TBF1 is a major molecular switch for plant growth-to-defense transition. Curr Biol 22, 103-112 (2012).

36. Z. T. Yang et al., The membrane-associated transcription factor NAC089 controls ER-stressinduced programmed cell death in plants. PLoS Genet 10, e1004243 (2014).

37. G. Heimberg, R. Bhatnagar, H. El-Samad, M. Thomson, Low Dimensionality in Gene Expression Data Enables the Accurate Extraction of Transcriptional Programs from Shallow Sequencing. Cell systems 2, 239-250 (2016).

38. S. Biswas et al., Tradict enables accurate prediction of eukaryotic transcriptional states from 100 marker genes. Nature communications 8, 15309 (2017).

39. Y. Donner, T. Feng, C. Benoist, D. Koller, Imputing gene expression from selectively reduced probe sets. Nat Methods 9, 1120-1125 (2012).

40. A. Dobin et al., STAR: ultrafast universal RNA-seq aligner. Bioinformatics 29, 15-21 (2013).

41. J. Jin et al., PlantTFDB 4.0: toward a central hub for transcription factors and regulatory interactions in plants. Nucleic Acids Res 45, D1040-d1045 (2017).

42. D. C. Montgomery, E. A. Peck, G. G. Vining, Introduction to linear regression analysis. Wiley series in probability and statistics (Wiley, Hoboken, NJ, ed. 5th, 2012), pp. xvi, 645 p.

43. J. Schafer, K. Strimmer, A shrinkage approach to large-scale covariance matrix estimation and implications for functional genomics. Stat Appl Genet Mol Biol 4, Article32 (2005).

44. P. Shannon et al., Cytoscape: a software environment for integrated models of biomolecular interaction networks. Genome Res 13, (2003). 
45. T. Z. Berardini et al., The Arabidopsis information resource: Making and mining the "gold standard" annotated reference plant genome. Genesis 53, 474-485 (2015).

46. Z. Gu, L. Gu, R. Eils, M. Schlesner, B. Brors, circlize Implements and enhances circular visualization in R. Bioinformatics 30, 2811-2812 (2014).

\section{FIGURES}

Figure 1. Building an Arabidopsis gene expression predictor. (A) The analysis workflow. (B) Principle component analysis of the 24545 RNA-Seq samples used for training the predictor model. PC, principle component. (C) Three predictor models trained with different combinations of leaf, root, and seed samples were tested for predicting performance on independent leaf $(n=500)$, root $(n=500)$, and seed $(n=500)$ samples. Shown are the average Pearson's correlation coefficients $(r)$ and normalized root mean square errors (NRMSE) between the predicted and actual transcriptomes. (D) Predictor models trained with different number of training samples were tested for their performance. Shown are the average $r$ (left) and NRMSE (right) between the predicted and actual transcriptomes for the training samples (black) and independent test samples (red, $\mathrm{n}=3000$ ).

Figure 2. The predictor's predicting performance on independent novel samples. (A) Two scatterplots showing the predicted and actual transcriptomes for two RNA-Seq samples derived from Arabidopsis root and shoot tissues. The two transcriptomes were generated in our lab to test the model's performance. (B) A boxplot showing the leave-one-out cross-validation (LOOCV) test result for samples from the SRA study SRP075604 (13). (C) LOOVC test results for 546 SRA studies with 10+ samples. For each study, average LOOCV test performance statistics ( $r$ and NRMSE) were calculated for all its samples, which were then used to generate the histograms showing the performance distribution for all 546 studies.

Figure 3. Gene co-expression modules identified via co-expression network analysis. (A) The 20 largest gene co-expression modules identified from the Arabidopsis GGM gene co-expression network. Dots represent genes, and co-expressed genes are linked by edges. Colors indicate module identities. Only the top 20 largest modules are shown due to space limit. (B - H) Subnetworks for selected gene co-expression modules that function in suberin (B), glucosinolate (C), flavonoid (D) biosynthesis, lateral root (E), xylem (F), secondary cell wall (G) development, and endoplasmic reticular stress response (H). The module id and represented enriched GO term (and its p-value) are shown for each module, with the genes possessing the represented GO term highlighted in red text.

Figure 4. Predictor TFs identified for genes and pathways. (A) A Chord diagram linking the gene CYP86B1 and its 37 predictor TFs. Colors indicate the p-value associated with the correspondent coefficients for the TF-target gene pairs extracted from the predictor, while the width of the links are proportional to the 
magnitude of the coefficient. (B - H) Chord diagrams linking the selected module's predictor TFs to their target genes within the module. The links are color-coded by TF genes, their left ends' colors indicate the p-values of the corresponding coefficients, while their widths are proportional to the coefficients' magnitude. Also indicated are the p-values for the enrichment of the TF's target genes within the module. Predictor TFs in red text are known regulators of the corresponding pathways. Due to space limit, only 1520 target genes were shown.

\section{SUPPLEMENTAL FIGURES AND TABLES}

Figure S1. Distribution of all partial correlation coefficients. A histogram showing the distribution of all pcors between 30860 genes. Only those gene pairs with pcors $>=0.045$ were used for GGM gene coexpression network construction.

Table S1. Significant co-expression gene pairs used for GGM gene co-expression network construction.

Table S2. Module identities for the genes within the co-expression network.

Table S3. Gene Ontology enrichment analysis results for the modules.

Table S4. Significant interacting TF-target gene pairs identified form the predictor.

Table S5. Predictor TFs identified for the gene co-expression modules. 
bioRxiv preprint doi: https://doi.org/10.1101/2020.04.07.029413; this version posted April 8, 2020. The copyright holder for this preprint (which was not certified by peer review) is the author/funder, who has granted bioRxiv a license to display the preprint in perpetuity. It is made available under aCC-BY-NC-ND 4.0 International license.

\section{Figure 1}

A

Model: $Y=X B+\varepsilon$

Training:

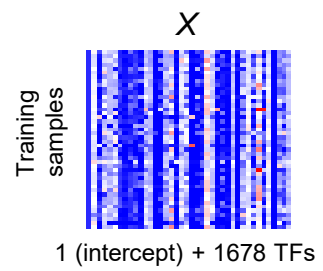

\section{Prediction:}

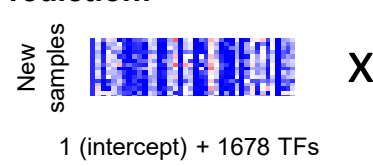

Inference:

\section{Expression leve}
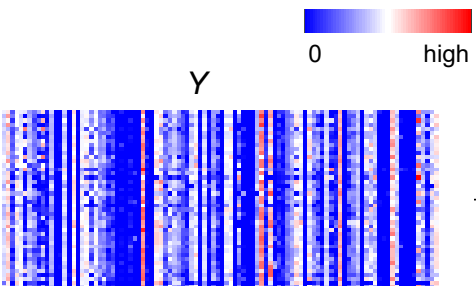

29182 target genes
B

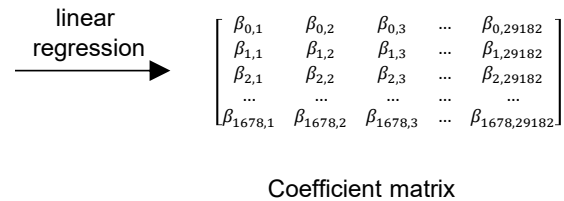

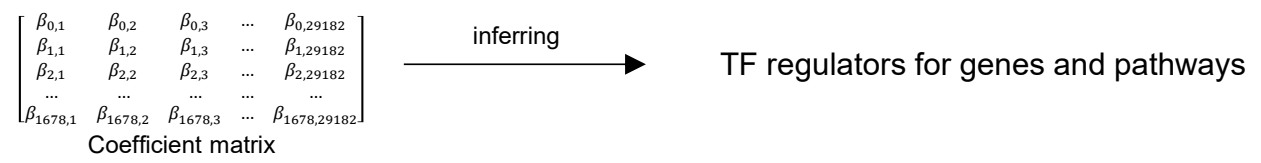

C

Predicted expression

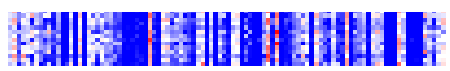

29182 target genes
B

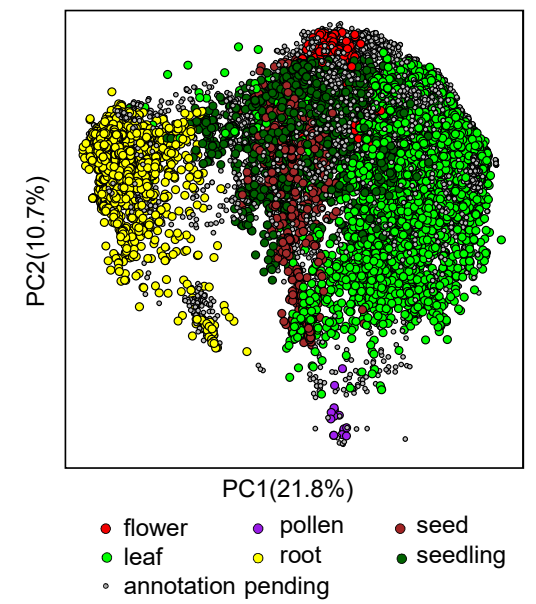

Model predicting performance on independent test samples

\begin{tabular}{|c|c|c|c|c|c|c|}
\hline \multirow{2}{*}{ Model (training data) } & \multicolumn{3}{|c|}{$\boldsymbol{r}$} & \multicolumn{3}{|c|}{ NRMSE } \\
\cline { 2 - 7 } & leaf & root & seed & leaf & root & seed \\
\hline I (7000 leaf samples) & 0.9863 & 0.9297 & 0.783 & 0.1158 & 0.2585 & 0.4913 \\
\hline II (7000 leaf, 1500 root samples) & 0.9869 & 0.9895 & 0.8117 & 0.1128 & 0.0996 & 0.4511 \\
\hline III (7000 leaf, 1500 root, 500 seed samples) & 0.9871 & 0.9896 & 0.9887 & 0.1119 & 0.0992 & 0.1069 \\
\hline
\end{tabular}

D
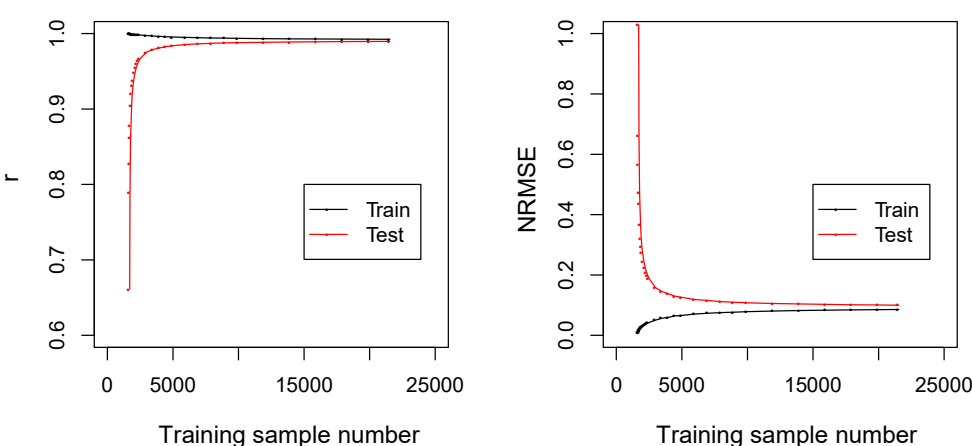
bioRxiv preprint doi: https://doi.org/10.1101/2020.04.07.029413; this version posted April 8, 2020. The copyright holder for this preprint (which was not certified by peer review) is the author/funder, who has granted bioRxiv a license to display the preprint in perpetuity. It is made available under aCC-BY-NC-ND 4.0 International license.

\section{Figure 2}

A

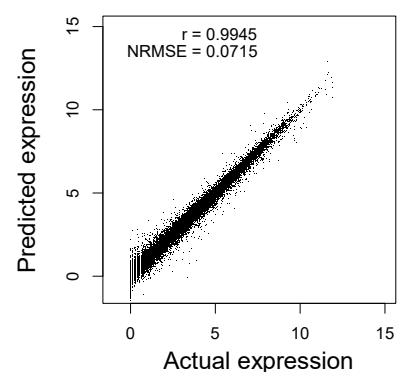

$\mathrm{C}$

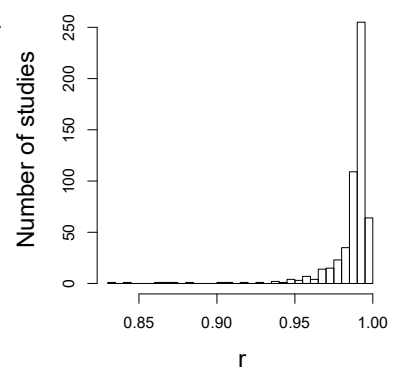

Shoot
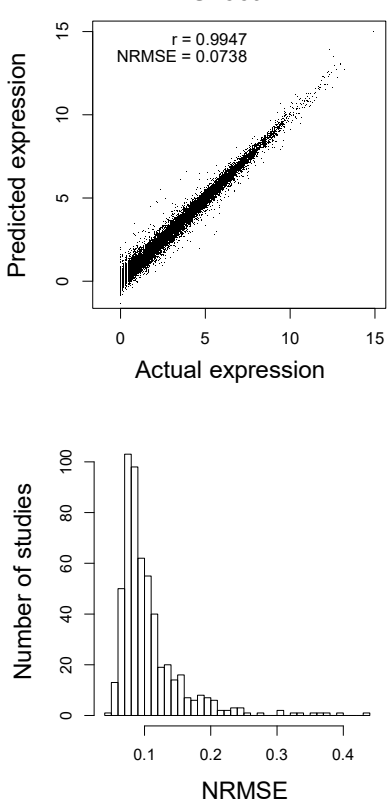

B

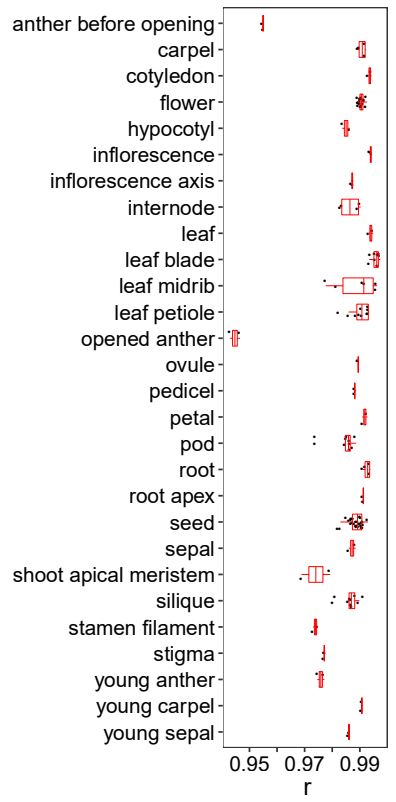


bioRxiv preprint doi: https://doi.org/10.1101/2020.04.07.029413; this version posted April 8, 2020. The copyright holder for this preprint (which was not certified by peer review) is the author/funder, who has granted bioRxiv a license to display the preprint in perpetuity. It is made available under aCC-BY-NC-ND 4.0 International license.

\section{Figure 3}
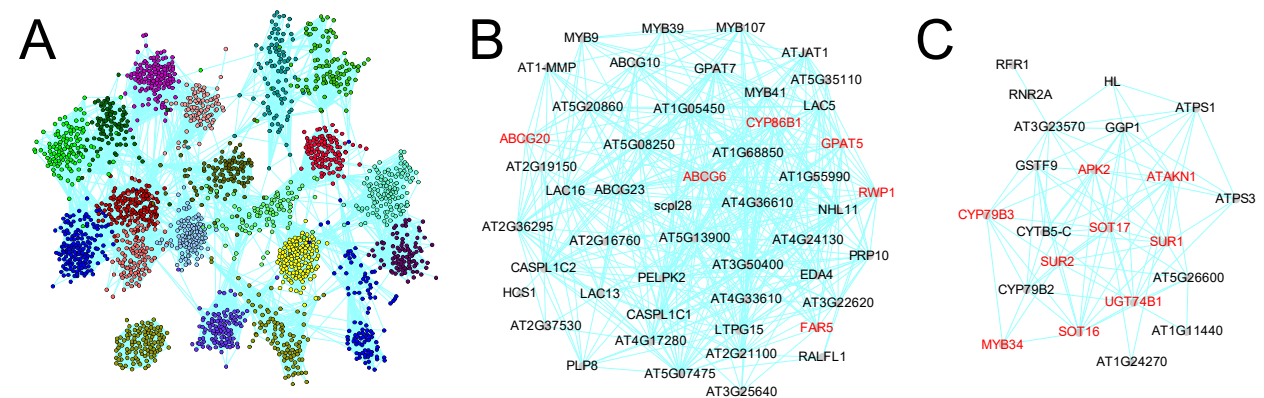

\#84 suberin biosynthesis process

$$
\text { \#272 glucosinolate biosynthetic }
$$

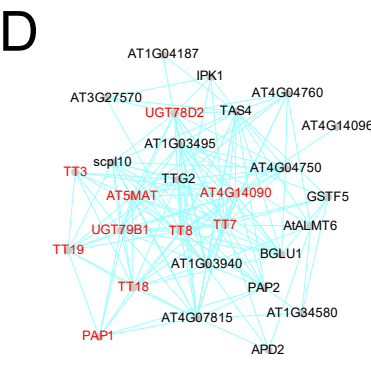
E
AT5G36960
AT2G22460
MTB56
AT3660670
SOK1

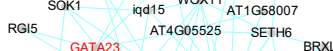 AT2G15590 GH3.1 LBD16 FL4 BRXL1 ATM1 PUCHI LBD17 MAKRA IAA11
AT3G15250 CYP78A10 LBD29 UCNL AT1G60010 TOLS2 AT1G64405$$
\text { LBD19 }{ }^{\text {IDL4 }} \text { AT5G67430 }
$$$$
\begin{aligned}
& \text { ATT29195 RT19 } \\
& \text { ZFP2 AT2G23060 AT1G75090 }
\end{aligned}
$$$$
\text { MES17 AT3G46800 }
$$

\#210 flavonoid biosynthetic process (1.02E-17)

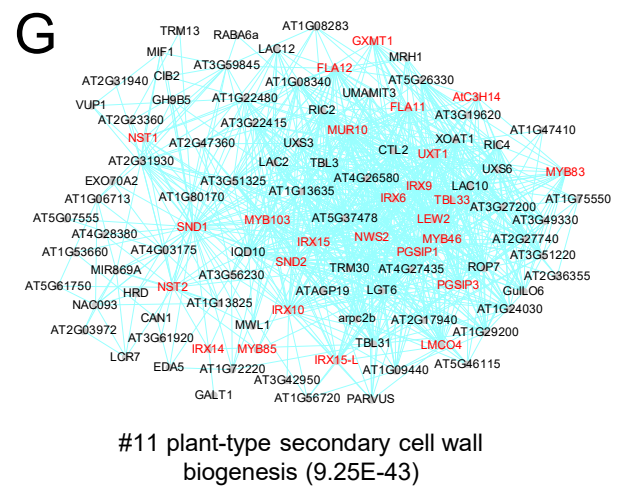

ral root development (1.86E-06)
F at2G40640 DTA4 aT5G01070 SMR113 VND5 LACE AT2G28410 AT4G11950 AT5G02640 $\begin{array}{llll}\text { AT1G14190 NHX4 AT1653163 } & \end{array}$ AT1G14190 NT1G23340 TRX1 PXC2 T5L1 VND3 AT4G22900 PXC3 SAMDC4 AT3G28420 MSL10 ACL5 $2 A \times 2$ AT3G10080 TMA30 ICU4 PIN1 AT1G10750 VND1 REV TDR ATHB8 AT1647655 AT1G70550 PHB PING ${ }_{\text {BHLH106 }}{ }_{\text {PID2 }}$ TMKL1 ${ }_{\text {AT1G47670 }}$ WAT1 1 BHLH106 PHV AT2G28660 AT5G62710 AT3G56770 \#65 xylem development (1.05E-14)

H

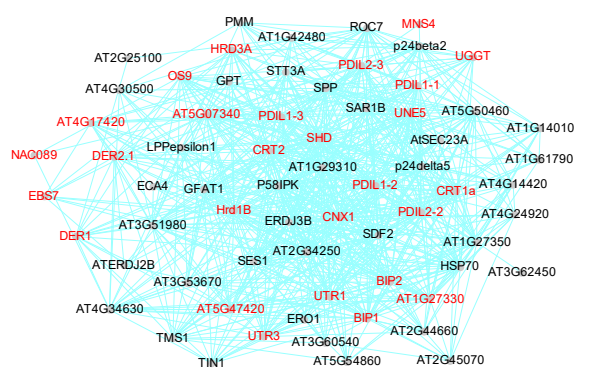

\#46 response to endoplasmic reticulum stress (3.96E-50) 
bioRxiv preprint doi: https://doi.org/10.1101/2020.04.07.029413; this version posted April 8, 2020. The copyright holder for this preprint (which was not certified by peer review) is the author/funder, who has granted bioRxiv a license to display the preprint in perpetuity. It is made available under aCC-BY-NC-ND 4.0 International license.

\section{Figure 4}

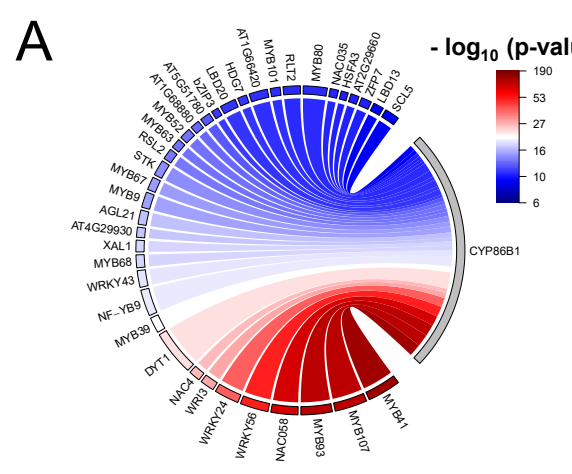

C

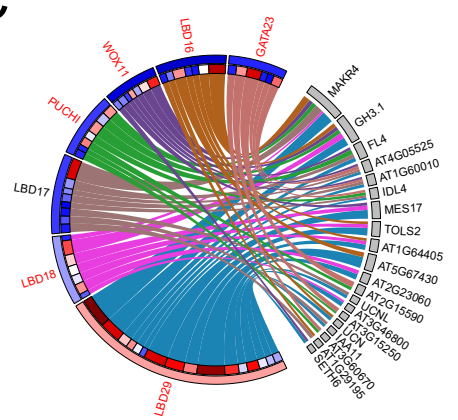

\#105 lateral root development

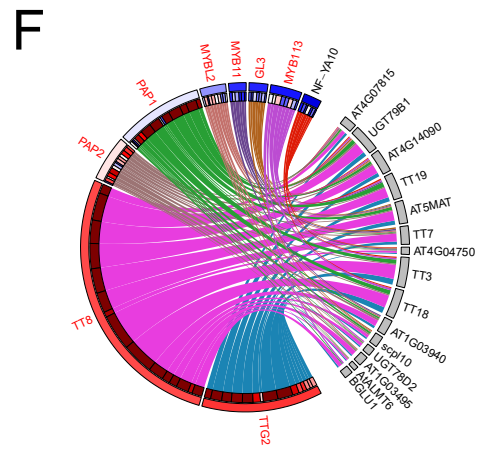

\#210 flavonoid biosynthesis

D

\section{G}
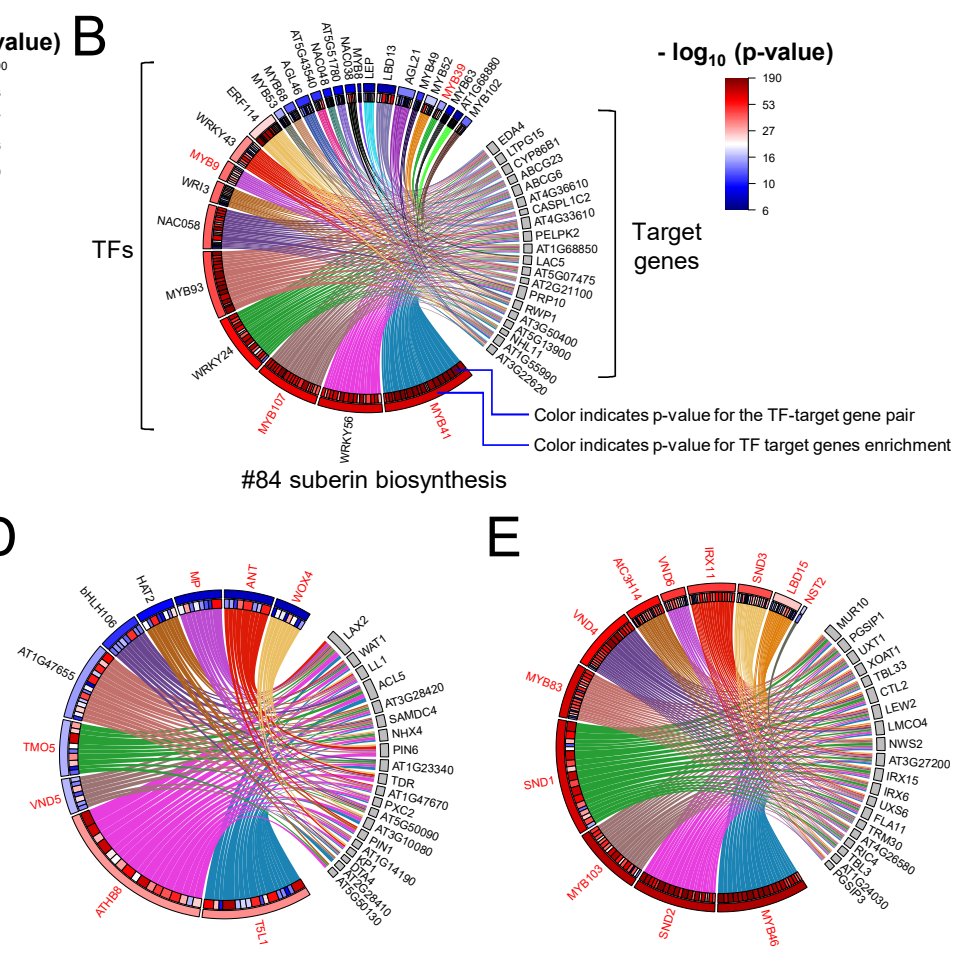

E

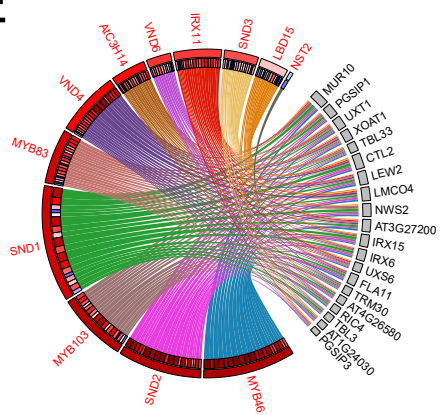

\#11 secondary cell wall biogenesis

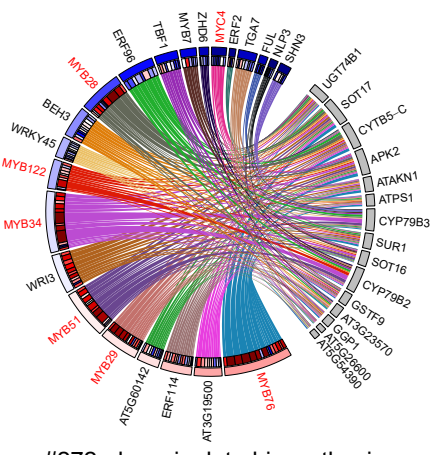

$\mathrm{H}$

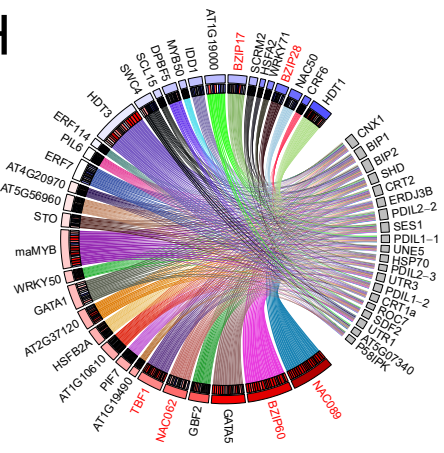

\#46 ER stress response 